\title{
A MODIFIED METHOD FOR POLARIMETRIC SAR CALIBRATION ALGORITHM
}

\author{
Lu Liao, Pingxiang Li, Jie Yang
}

The State Key Laboratory of Information Engineering in Surveying, Mapping and Remote Sensing of Wuhan University, P. R. China, liaoluwhuwman@163.com

\section{Commission VII WGVII/6}

KEY WORDS: Channel imbalance, Calibration, Co-polarization, Corner reflector, Crosstalk, Distortion

\begin{abstract}
:
Present fully polarimetric synthetic aperture radar (SAR) systems often update calibration techniques to further enhance the accuracy to the polarimetric data. In this paper, we propose a modified method to estimate the value of crosstalk based on the corrected observed value. Since Ainsworth calibration algorithm firstly set the value of $k$ to be one. And the value of $k$ relates to the copolarization channel imbalance. We consider the effects of value of $k$ and analyze it. Through comparison to crosstalk results between the stimulated parameters and the estimated parameters, we assume high co-polarization channel imbalance will be obviously to affect crosstalk results. Then, used covariance observation value of the initial value of $k$ rewrites the model to solve related parameters. And crosstalk parameter is calculated by the same iterative method. To verify the effect of the modified calibration method, this letter compares the accuracy of the two methods using the simulated polarimetric SAR data and Chinese airborne X-band polarimetric SAR data. The results confirm that the modified method tends to get more accurate crosstalk results.
\end{abstract}

\section{INTRODUCTION}

XSAR system is developed by the China Electronics Technology Group Corporation 38 department. The radar system is placed on China "Y-7" airborne platform and the mean flight velocity is nearly $120 \mathrm{~m} / \mathrm{sec}$, at $5000 \mathrm{~m}$ altitude. The radar incidence angle could be adjusted from 37degree to 45 degree and the resolution are $0.1 \mathrm{~m}, 0.4 \mathrm{~m}$ respectively (azimuth resolution and slant resolution)[1].

Fully polarimetric SAR data calibration is important to ensure accurate extraction of geophysical parameters, such as, soil moisture, surface roughness, and biomass [2]. The calibration algorithms proposed by van Zyl in [3] and the more general approach proposed by Quegan in [4] are today standards for phase and cross-talk calibration of polarimetric data. In 2006 Ainsworth proposed a posteriori method imposing only the weakest of constraints, scattering reciprocity, on the polarimetric data [5]. Thus, due to only reciprocity assumption which implies that the two cross-polarization channels, HV and $\mathrm{VH}$, are identical, Ainsworth algorithm should have wider application scope than Quegan algorithm, and present more accurate results for complex surface features in practice.After lots of research and calibration experiments, we find that the estimation of crosstalk parameters by Ainsworth algorithm is easy to affected by the value of $k$. When $k$ is more close to one, the accuracy of crosstalk is better. But if $k$ is far to one, the accuracy of crosstalk is unstable. In this letter, we propose a modified method, based on the Ainsworth algorithm. The processing of this new method is as follows: 1) Estimate the imbalance ratio $\alpha, 2)$ Estimate the imbalance ratio $k$ and

produce $\left.\left[\Sigma^{\prime}\right], 3\right)$ Estimate $A$ and $B .4$ ) Estimate the correction values of these four estimations, and update the crosstalk parameters. Since the value of $k$ is no assumption (set $k=1$ ) in the process of the parameter estimation, the modified calibration method can accurately solve crosstalk parameters, even if the channel imbalance is high. The simulated and calibrated data for Chinese airborne XSAR system are used to validate the effectiveness of modified calibration method. The experimental results demonstrate that the modified method proves to be more accurate for crosstalk estimation.

\section{POLARIMETRIC CALIBRATION ALGORITHM}

For calibration procedure, a model that relates the observed polarimetric signals to the desired (true) calibrated signals is needed. In principle, this model incorporates parameters for crosstalk, channel imbalance, system noise, systematic peculiarities, etc. In practice we employ a standard model that relates the transmitted horizontally $(\mathrm{H})$ and vertically $(\mathrm{V})$ polarized signals to the polarized received signals. Basic equation (1):

$$
\begin{aligned}
& O=[R][S][T]+N \\
& =\left[\begin{array}{ll}
r_{11} & r_{12} \\
r_{21} & r_{22}
\end{array}\right]\left[\begin{array}{ll}
S_{H H} & S_{H V} \\
S_{V H} & S_{V V}
\end{array}\right]\left[\begin{array}{ll}
t_{11} & t_{12} \\
t_{21} & t_{22}
\end{array}\right]+\left[\begin{array}{ll}
n_{H H} & n_{H V} \\
n_{V H} & n_{V V}
\end{array}\right]
\end{aligned}
$$

Where $\boldsymbol{O}$ is the observed scattering matrix, $\boldsymbol{S}$ is the Scattering matrix, $\boldsymbol{R}$ and $\boldsymbol{T}$ are receive and transmit distortion matrices, respectively, $\boldsymbol{N}$ is the noise matrix.

\subsection{Calibration equations}

Ainsworth proposed an a posteriori means to calibrate both cross talk between channels and imbalances in the channel gains employing only the observed polarimetric SAR data without invoking reflection symmetry. Converting the scattering matrix to a vector format and rewriting the previous model yields:

$$
\left[\begin{array}{c}
O_{H H} \\
O_{H V} \\
O_{V H} \\
O_{V V}
\end{array}\right]=\left[\begin{array}{cccc}
k a & v a^{-1} & w a & v w k^{-1} a^{-1} \\
z k a & a^{-1} & w z a & w k^{-1} a^{-1} \\
u k a & u v a^{-1} & a & v k^{-1} a^{-1} \\
u z k a & u a^{-1} & z a & k^{-1} a^{-1}
\end{array}\right]\left[\begin{array}{l}
S_{H H} \\
S_{H V} \\
S_{V H} \\
S_{V V}
\end{array}\right]=[M]\left[\begin{array}{c}
S_{H H} \\
S_{H V} \\
S_{V H} \\
S_{V V}
\end{array}\right]
$$


values of $u, v, w$ and $z$ parameterize the channel crosstalk. The definitions are $k=r_{11} / r_{22}, \alpha=r_{22} t_{11} / r_{11} t_{22}, u=r_{21} / r_{11}, v=t_{21} / t_{22}, w$ $=r_{12} / r_{22}$ and $z=t_{12} / t_{11}$. An overall gain factor, $r_{22} t_{22}$, has been dropped, so absolute radiometric calibration is not considered. All of these parameters are complex. Rewriting in terms of the covariance matrices the calibration (2) becomes

$$
[C]=[O][O]^{\dagger} \propto[M][S][S]^{\dagger}[M]^{\dagger}=[M][\Sigma][M]^{\dagger}
$$

Where $[M]$ is the $4 \times 4$ calibration matrix and the observed covariance is given as $[C]$. The $\dagger$ symbol denotes the Hermitian conjugate. Now enforcing equality of $S_{\mathrm{hv}}$ and $S_{\mathrm{vh}}$ implies several constraints on the $[\Sigma]$ covariance matrix, (4). By construction $[\Sigma]$ is Hermitian.

$$
\Sigma_{\mathrm{HVHV}}=\Sigma_{\mathrm{VHVH}} \quad \Sigma_{\mathrm{HVHV}}=\Sigma_{\mathrm{VHHV}}
$$

$\operatorname{Im}\left(\Sigma_{\mathrm{VHVH}}\right)=0 \quad \Sigma_{\mathrm{HVHH}}=\Sigma_{\mathrm{VHHH}} \quad \Sigma_{\mathrm{HVVV}}=\Sigma_{\mathrm{VHVV}}$

Thus the corresponding set of complex conjugate equations do not provide independent information. We require a model to describe the proper form of the calibrated covariance matrix.

\subsection{Calibration model}

The general form of the calibrated covariance matrix in the linear basis, assuming the scattering is reciprocal, is

$$
[\Sigma]=\left[\begin{array}{cccc}
\Sigma_{\mathrm{HHHH}} & A^{*} & A^{*} & \Sigma_{\mathrm{HHVV}} \\
A & \beta & \beta^{\prime} & B \\
A & \beta^{\prime} & \beta & B \\
\Sigma_{\mathrm{VVHH}} & B^{*} & B^{*} & \Sigma_{\mathrm{vVVV}}
\end{array}\right]
$$

Where * denotes complex conjugation. Since reciprocity implies that the two cross-polarization channels, $\mathrm{HV}$ and $\mathrm{VH}$, are identical, one requires for calibrated data that they and all of their correlations are in fact identical. This form of the covariance matrix is very general, permitting nonzero helicities, orientation angles, etc. The observed polarimetric imagery determines the estimates of $A$ and $B$. Given $A$ and $B$, the calibration model (5) provides three real and four complex equations to determine the six complex numbers $k, \alpha, u, v, w, z$ and one real number $\eta=\beta-\beta^{\prime}$, the system noise.

$$
\begin{aligned}
& {\left[\Sigma^{\prime}\right]=[G]^{-1}[C][G]^{-1}}
\end{aligned}
$$

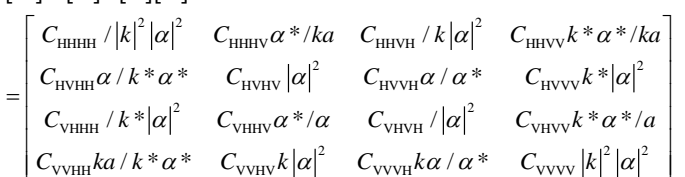

Model (6) and Equation (3) are linearized around the current values of $k, \alpha, u, v, w$ and $z$. The solution of this set of complex linear equations determines the updated values of $u, v, w$ and $z$. New estimate of $\alpha$ is made with the iteration repeats. Thus the non-linear solution for the calibration parameters is solved by iteration. The value of $k$ is equal to one in this process. The modified $[\Sigma$ '] by Ainsworth is as follows:

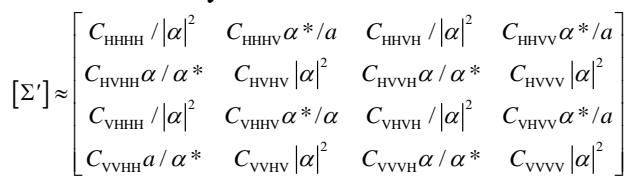

\subsection{Influence of $k$}

The values of $k$ and $\alpha$ determine the relative gains and phase delays between the polarimetric channels. Normally the estimates for $\mathrm{A}$ and $\mathrm{B}$ are independent of $k$. By default, Ainsworth set $k$ equal to one. The $k$ estimation and correction typically applies only as a last step. However, we can see that the value of $k$ directly affect to $\left[\Sigma^{\prime}\right]$ from (6). The elements of $[\Sigma$ '] determine $\mathrm{A}$ and $\mathrm{B}$ in (8).

$$
\begin{aligned}
& A=\left(C_{\mathrm{HVHH}} \alpha / \alpha^{*}+C_{\mathrm{VHHH}} /|\alpha|^{2}\right) / 2 k^{*}=\left(\Sigma_{\mathrm{HVHH}}^{\prime}+\Sigma_{\mathrm{VHHH}}^{\prime}\right) / 2 \\
& B=\left(C_{\mathrm{HVVV}}|\alpha|^{2}+C_{\mathrm{VHVV}} \alpha^{*} / a\right) k^{*} / 2=\left(\Sigma_{\mathrm{HVVV}}^{\prime}+\Sigma_{\mathrm{VHVV}}^{\prime}\right) / 2
\end{aligned}
$$

The crosstalk parameters are derived by solving a set of linear equations defined by (9)

$$
\mathrm{X}=[\zeta][\delta]+[\tau][\delta] *
$$

Where $[\delta]$ defines the incremental changes to crosstalk coefficients, and the remaining variables are defined as follows:

$$
\begin{gathered}
{[X]=\left[\begin{array}{c}
\Sigma_{\mathrm{HVHH}}^{\prime}-A \\
\Sigma_{\mathrm{VHH}}^{\prime}-A \\
\Sigma_{\mathrm{HVVV}}^{\prime}-B \\
\Sigma_{\mathrm{VHVV}}^{\prime}-B
\end{array}\right]=\frac{1}{2}\left[\begin{array}{l}
\Sigma_{\mathrm{HVHH}}^{\prime}-\Sigma_{\mathrm{VHHH}}^{\prime} \\
\Sigma_{\mathrm{VHHH}}^{\prime}-\Sigma_{\mathrm{HVHH}}^{\prime} \\
\Sigma_{\mathrm{HVVV}}^{\prime}-\Sigma_{\mathrm{VHVV}}^{\prime} \\
\Sigma_{\mathrm{VHVV}}^{\prime}-\Sigma_{\mathrm{HVVV}}^{\prime}
\end{array}\right]} \\
{\left[\begin{array}{c}
u \\
v \\
w \\
z
\end{array}\right]=\left[\begin{array}{l}
u_{0} \\
v_{0} \\
w_{0} \\
z_{0}
\end{array}\right]+\left[\begin{array}{c}
\delta_{u} \\
\delta_{v} \\
\delta_{w} \\
\delta_{z}
\end{array}\right]}
\end{gathered}
$$

The value of $k$ directly determines $u, v, w, z$ from (10) and (11) without affecting $\alpha$. So the inaccurate value of $k$ would get the inaccurate crosstalk parameters. The step that directly set $k$ equal to one help the method to achieve a unique solution but are inconsistent with the actual crosstalk condition.

\section{MODIFIED METHOD}

\subsection{Iteration scheme}

The first two steps of the Ainsworth iteration scheme is as follows: 1) Estimate the imbalance ratio $\alpha$, and produce $[\Sigma], 2$ ) estimate $A$ and $B$. To obtain more reasonable results, we suggest that the method would firstly solve the values of $k$. The first three steps of we proposed method is as follows: 1) Estimate the imbalance ratio $\alpha, 2)$ Estimate the imbalance ratio $k$ and produce $\left.\left[\Sigma^{\prime}\right], 3\right)$ Estimate $A$ and $B$. To obtain rapid convergence of the iterated solution, $k$ is no longer involved in iteration after produced $[\Sigma$ ']. The values of $k$ is more reasonable than set $k=1$. But this value is not the most accurate without crosstalk calibration. In the last step, we estimate the most accurate value of $k$ to replace the initial value of $k$ after completing cross-polarization channel imbalance and crosstalk calibration.

\subsection{Scheme of solving $k$}

The solving $k$ is relatively troublesome. Without additional information, the value of $k$ is indeterminate. The direct effect of $k$ is on the co-polarization returns and their correlations. Traditionally, an in-scene trihedral corner reflector fixes both the phase and amplitude of $k$ [6]-[11]. Alternatively, either theoretical or empirical models of presumed scattering mechanisms in the scene can be employed to set the $S_{\mathrm{HH}} / S_{\mathrm{VV}}$ ratio. Although the observed value contain untrue power from crosstalk effect. As we all know, power from crosstalk effect plays a relatively small role as compared to total power. Without crosstalk calibration, we assume that $u, v, w, z$ are zero. The initial value of $k$ is directly estimated by trihedral corner reflector or some empirical $S_{\mathrm{HH}} / S_{\mathrm{VV}}$ ratio. 


\section{EXPERIMENTAL RESULTS}

XSAR system has accomplished several flight plans from 2009 to 2010 in Hainan province and this paper experimental data set is obtained in February, 2010. The test suit locates on Lingshui city and the area center position is NL $18^{\circ} 28^{\prime} 55^{\prime \prime}$, EL $109^{\circ} 58^{\prime} 54^{\prime \prime}$.

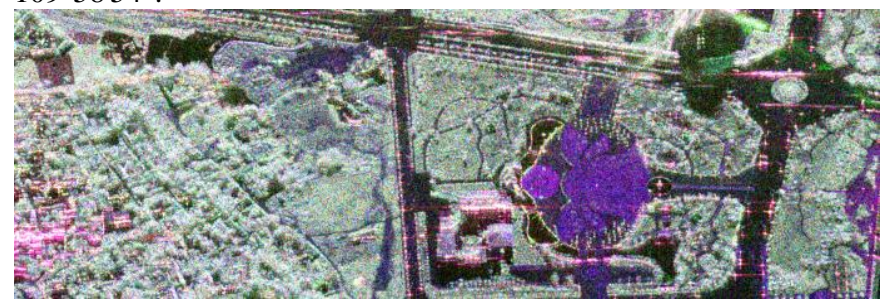

Figure 1. RGB image in pauli basis about Chinese airborne Xband polarimetric SAR after calibration. The top row is the nearest range, and the bottom row is the farthest range.

One trihedral corner reflector is the relatively bright pixels in RGB image, inside the red box, near the center part. The area is complex and includes a variety of natural and artificial targets, such as buildings, houses, fields, trees, bare ground and so on.

Based on the calibrated data, assuming some distortion parameters, we generate the simulated data, and then use different methods to solve these parameters and compare the accuracy of the methods. To further compare the accuracy of the calibration methods by different value of $k$ affect, we simulate a set of distorted data using real airborne X-band polarimetric SAR data. We assume that the amplitudes of $u, v, w, z$ are 0.005 , the amplitudes of $\alpha$ is 0.8 . The amplitudes of $k$ are respectively set to be $0.5,1$ and 1.5 .

\subsection{Corner reflector}

To obtain more reasonable results, the initial value of $k$ is estimated using the response from a trihedral corner reflector [3]. As we all know, an ideal trihedral corner reflector will show theoretical co-polarization and cross-polarization signature. The value of $k$ is estimated by $S_{\mathrm{HH}} / S_{\mathrm{VV}}=1$. Fig. 1 gives the copolarization signature response of simulated data set to be 0.5 and 1.5 , respectively

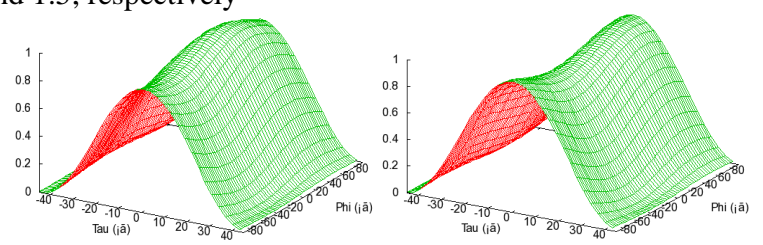

Figure 2. Co-polarization signature of trihedral corner reflector. (Left:Set to be 0.5, Right: Set to be 1.5)

Figure 2 shows the measured co-polarization signatures of the comer reflectors used simulated data, respectively set $\mathrm{k}$ to be 0.5 and 1.5. It is clear that the two signatures show noticeable distortion. After calculation, the estimated respective values of $k$ are 0.4754 and 1.6142 . The two values are very close to the simulated values.

In order to compare calibration methods by the original and the modified, the results are shown by Cross-polarization signature in Figure 3.

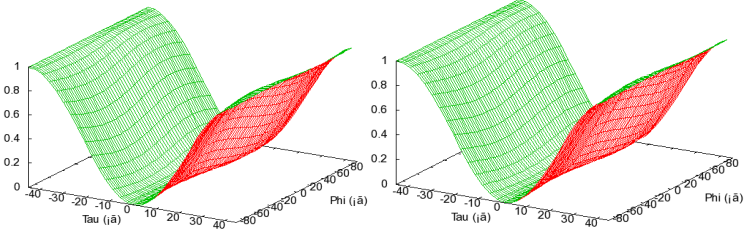

(a)

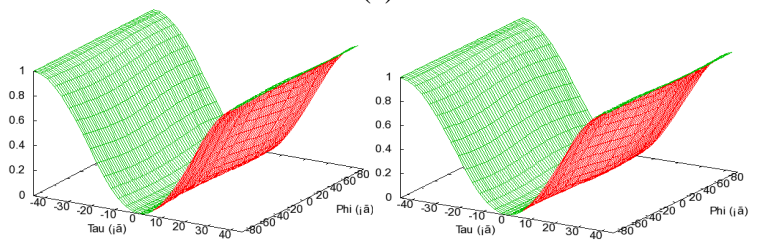

(b)

Figure 3. Cross-polarization signature of trihedral corner reflector. (Left: Set to be 0.5, Right: Set to be 1.5, (a): The original method, (b): The proposed method)

Figure3 (a) and (b) are the cross-polarization signatures after original and the modified method calibration, respectively set $\mathrm{k}$ to be 0.5 and 1.5. After cross-channel imbalance and crosstalk calibration, we can see that the modified method has better cross-polarization signature of than the original method.

\subsection{RGB image}

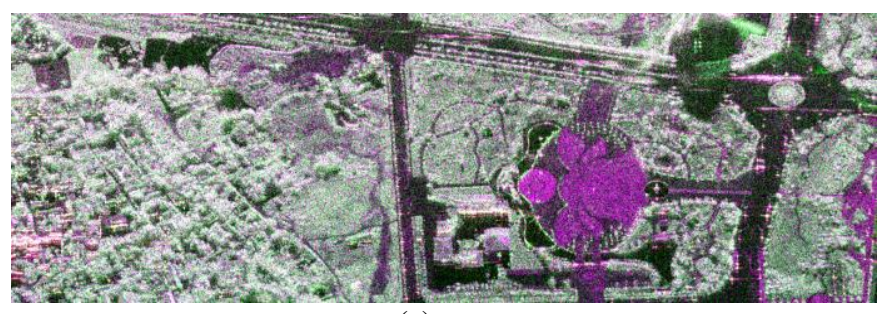

(a)

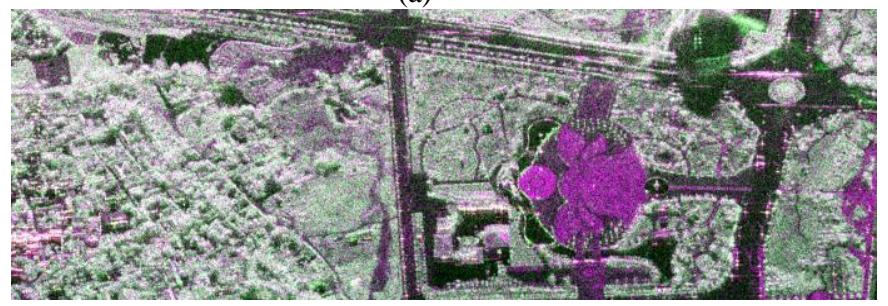

(b)

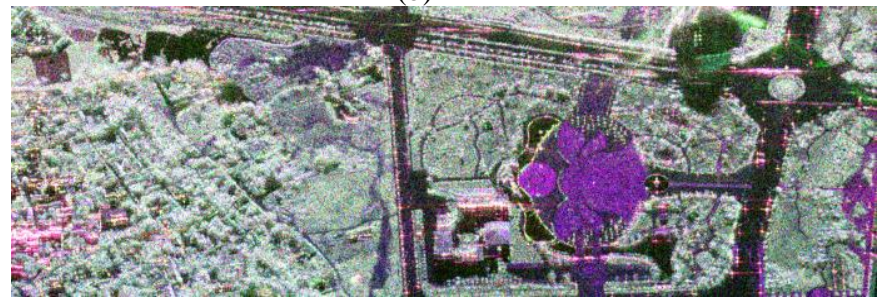

(c)

Figure 4. RGB image in Pauli basis before and after simulated data calibration, set $k=0.5$. (a) RGB image before calibration. (b)

Calibrated image using the original method. (c) Calibrated image using the modified method.

Figure 4 shows Chinese airborne X-band polarimetric SAR data before and after calibration, rendered in Pauli basis. From Figure 4 (a) and (b) we can see that the original image is overall reddish, including ceramic tile area (odd scattering) and bare soil (odd scattering); after the original calibration, the corresponding areas have no obvious improvement. Using the modified method, ceramic tile area and bare soil is closer to the 
truth from Figure1. Their colour is better to correspond to the scattering mechanism.

\subsection{Crosstalk}

The value of $k$ directly determines $u, v, w, z$. The inaccurate value of $k$ would get the inaccurate crosstalk results, and the results that directly set $k$ equal to one are presented in Table 1 . The amplitudes of $k$ are respectively set to be $0.5,1$ and 1.5 . We can see that if $k=1$, the crosstalk results is closer to the actual values. But if $k=0.5$ or $k=1.5$, the crosstalk results are significantly different from the actual values after Ainsworth method calibration.

\begin{tabular}{|c|ccccc|}
\hline$k(\mathrm{set})$ & $k$ & $u=0.005$ & $v=0.005$ & $w=0.005$ & $z=0.005$ \\
\hline 0.5 & 1 & 0.0012 & 0.0108 & 0.0108 & 0.0012 \\
\cline { 1 - 1 } 1 & 1 & 0.0023 & 0.0053 & 0.0053 & 0.0023 \\
\cline { 1 - 1 } & 1 & 0.0034 & 0.0036 & 0.0036 & 0.0034 \\
\hline
\end{tabular}

Table 1. Ainsworth calibration results Using the modified method, the crosstalk results are presented in Table 2. Compare Table 2 to Table 1, we can see that crosstalk results are better to correspond to the actual values after the modified method calibration.

\begin{tabular}{|l|lllll|}
\hline$k$ (set) & $k$ (solve) & $u=0.005$ & $v=0.005$ & $w=0.005$ & $z=0.005$ \\
\cline { 1 - 2 } & 0.4754 & 0.0036 & 0.0072 & 0.0072 & 0.0036 \\
\cline { 1 - 1 } 1.5 & 1.6142 & 0.0039 & 0.0056 & 0.0056 & 0.0039 \\
\hline
\end{tabular}

Table 2. The modified method calibration results

\section{CONCLUSION}

In this letter, Ainsworth algorithm for crosstalk calibration of polarimetric SAR data has been modified. An initial value of $k$ is introduced based on the empirical $S_{\mathrm{HH}} / S_{\mathrm{VV}}$ ratio. Then calibration parameters are estimated based on the corrected observed value. This modified method is tested for the X-band China domestic polarimetric data. The improvement is demonstrated on the calibrated response of a corner reflector, the RGB image in pauli basis, and comparison to simulate parameters. The improvement is mainly attributed to the initial value of $k$. The modified method solves the original method's problem in the case of the high co-channel imbalance. The experimental results confirm that the modified method can solve crosstalk parameters more accurately.

\section{REFERENCES}

[1] P.X. Li, L. Shi, J. Yang, L.J. Lu, 2011. Assessment of Polarimetric and Interferometric Image Quality for Chinese Domestic X-Band Airborne SAR System, in Proc. ISIDF, pp.1-8.

[2] Freeman, A., 1992. SAR calibration: An overview. IEEE Transaction on Geoscience and Remote Sensing, vol. 30, pp.1107-1121.

[3] J.J. van Zyl., 1990. Calibration of polarimetric radar images using only image parameters and trihedral corner reflector responses. IEEE Transaction on Geoscience and Remote Sensing, 28, pp.337-348.

[4] Quegan. S., 1994. A unified algorithm for phase and crosstalk calibration of Polarimetric data theory and observations, IEEE Transaction on Geoscience and Remote Sensing, 32(1), pp.89-99.
[5] Ainsworth,T. Ferro-Famil,L. L. and Lee, J.S., 2006. Orientation angle preserving a posteriori Polarimetric SAR Calibration. IEEE Transaction on Geoscience and Remote Sensing, vol.44, no.4, pp.994-1003.

[6] J.D. Klein and A. Freeman, 1991. Quadpolarization SAR calibration using target reciprocity. J. Electromagn. Waves Propagat., vol. 5, pp.735-751.

[7] M.W. Whitt, F.T. Ulaby, P. Polatin, and V. V. Liepa, 1991. A general polarimetric radar calibration technique. I IEEE Transaction on Geoscience and Remote Sensing,vol. 39, no.1, pp.62-67.

[8] J.-R. J. Gau and W.D. Burnside, 1995. New polarimetric calibration technique using a single calibration dihedral. Proc. Inst. Elect. Eng., Microw. Antennas Propagat., vol. 142, pp.19-25.

[9] J. J. van Zyl, 1990. Calibration of polarimetric radar images using only image parameters and trihedral corner reflector responses. IEEE Transaction on Geoscience and Remote Sensing, vol.28, no.3, pp.337-348.

[10] K. Sarabandi, L.E. Pierce, M.C. Dobson, F.T. Ulaby, J.M Stiles, T.C. Chiu, R. De Roo, R. Hartikka, A. Zambetti, and A. Freeman, 1995. Polarimetric calibration of SIR-C using point and distributed targets, IEEE Transaction on Geoscience and Remote Sensing, vol.33, no.4, pp.858-866.

[11] H. A. Zebker, J. J. van Zyl, S. L. Durden, and L. Norikane, 1991.Calibrated imaging radar polarimetry: Technique, examples, and applications. IEEE Transaction on Geoscience and Remote Sensing, vol.29, no.6, pp.942-961.

\section{ACKNOWLEDGEMENTS}

The research summarized in this paper was supported by funding from National High Technology Research and Development Program ("863 Program") (Grant No.2011AA120404) and Land Remote Sensing Application Research On Multidimensional Microwave Imaging (No.60890074). The authors would like to thank the CETC38 for the data set providing and valuable recommendations with respect to this paper. 